\title{
Fibroblasts restrain drug sensitivity
}

MAFs are
responsible
for an initial
phase of
insensitivity to
PLX4720

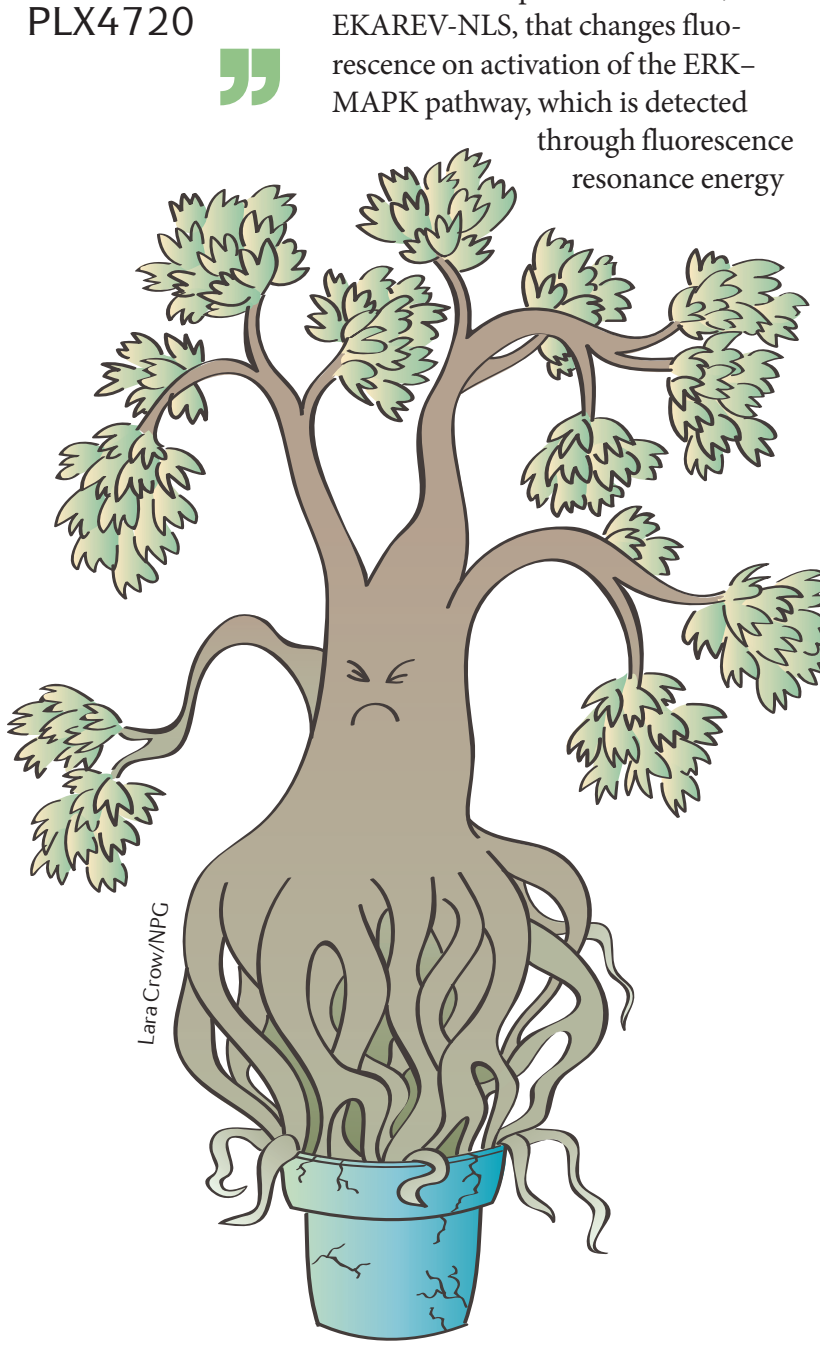

Hirata et al. found that two BRAF-V600E-mutant mouse melanoma cell lines were sensitive to the BRAF-V600E inhibitor PLX4720 in vitro, but when grown as syngeneic tumours in vivo, they were no longer sensitive. The authors engineered these cells to express a biosensor, EKAREV-NLS, that changes fluorescence on activation of the ERKwhich is detected

.

Non-cell-autonomous mechanisms of melanoma resistance to BRAF-V600E inhibitors have been proposed, but it is not clear how the tumour microenvironment might drive resistance. line conferred resistance to PLX4720 and induced invasive characteristics in the melanoma cells. This indicates that MAFs are responsible for an initial phase of insensitivity to PLX4720.

This adaptation to PLX4720 occurred too quickly to be genetic; therefore, to investigate the mechanism of this initial phase of drug resistance, the authors engineered MAFs to express the EKAREV-NLS biosensor and mCherry to distinguish them from melanoma cells. Imaging of spheroid cultures treated with PLX4720 revealed that ERK-MAPK pathway activity increased in the MAFs, but decreased in the melanoma cells. PLX4720 slightly increased MAF proliferation in these spheroids, and collagen gel contraction assays showed that the drug increased collagen remodelling by MAFs; normal fibroblasts were unaffected by PLX4720. Gene expression analysis revealed that MAFs upregulated expression of many matrix molecules, such as thrombospondin 1 (THBS1) and tenascin C (TNC), as well as platelet-derived growth factor receptor- $\alpha$ in response to PLX4720. Moreover, the authors found that culturing melanoma cells on stiff matrices coated with fibronectin, THBS1 and TNC, in the absence of MAFs, was sufficient to abrogate responses to PLX4720.

The authors also found evidence of focal adhesion kinase (FAK) activation upon treatment of melanoma and MAF co-cultures with PLX4720; inhibition of FAK and PLX4720 prevented spheroid co-cultures from reactivating ERK-MAPK in vitro, and this combination was effective in vivo.

Next, the authors turned to xenografts of PLX4720-sensitive human melanoma cells. They found that substantial residual disease remained after treatment with PLX4720 and that these cells exhibited ERKMAPK reactivation. Moreover, these residual cells were accompanied by matrix remodelling. Isolated residual melanoma cells were still sensitive to PLX4720, indicating that they had not yet acquired genetic resistance. Combined PLX4720 and FAK inhibition of established PLX4720-sensitive human melanoma xenografts resulted in prolonged ERK inactivation, reduced melanoma cell proliferation and significantly reduced tumour burden. FAK inhibition plus PLX4720 also prolonged the control of a therapy-naive patient-derived melanoma xenograft.

Therefore, MAFs prevent the effects of PLX4720 on melanoma cells, which can then continue to divide, thus potentially allowing for the selection of melanoma cells that are genetically resistant to PLX4720.

Gemma K. Alderton

ORIGINAL RESEARCH PAPER Hirata, E. et al. Intravital imaging reveals how BRAF inhibition generates drug-tolerant microenvironments with high integrin $\beta 1 /$ FAK signaling. Cancer Cell 27, 574-588 (2015) 